\title{
(c) OPEN ACCESS \\ Comprehensive analysis of intra-arterial treatment for acute ischemic stroke due to cervical artery dissection
}

\author{
Judd Jensen, ${ }^{1}$ Kristin Salottolo, ${ }^{2}$ Donald Frei, ${ }^{1,3}$ David Loy, ${ }^{1,3}$ Kathryn McCarthy, \\ Jeffrey Wagner, ${ }^{1}$ Michelle Whaley, ${ }^{1}$ Richard Bellon, ${ }^{1,3}$ David Bar-Or ${ }^{2}$
}

'Department of Neurology, Swedish Medical Center, Englewood, Colorado, USA 2Trauma Research LLC, Swedish Medical Center, Englewood, Colorado, USA

${ }^{3}$ Radiology Imaging Associates PC, Englewood, Colorado, USA

Correspondence to

Dr D Bar-Or, Trauma Research Department, Swedish Medical Center, 501 E Hampden Ave, Rm 4-454, Englewood, CO 80113, USA;

dbaror@ampiopharma.com

Received 28 March 2016 Revised 18 May 2016 Accepted 20 May 2016 Published Online First 10 June 2016
CrossMark

To cite: Jensen J,

Salottolo K, Frei D, et al.

I Neurolntervent Surg

2017;9:654-658.
ABSTRACT

Objective The safety and efficacy of intra-arterial treatment (IAT) in patients with acute ischemic stroke (AIS) due to cervical artery dissection (CeAD) has not been formally studied. The purpose of this study was twofold: first, describe a large series with CeAD treated with IAT; second, analyze outcomes with CeAD receiving IAT versus (a) CeAD not treated with IAT, (b) CeAD receiving intravenous thrombolysis (IVT) alone, and (c) non-CeAD mechanism of AIS receiving IAT.

Design Demographics, clinical characteristics, treatment, and outcomes were summarized for all CeAD patients treated with IAT from January 2010 to May 2015. Outcomes included favorable 90 day modified Rankin Scale (mRS) score of 0-2, symptomatic intracerebral hemorrhage $(\mathrm{s} I \mathrm{CH})$, recanalization (Thrombolysis in Cerebral Infarction 2b-3), procedural complications, and mortality. Outcomes were analyzed with $\chi^{2}$ tests and multivariate logistic regression.

Results There were 161 patients with CeAD: 24 were treated with IAT and comprised our target population. Dissections were more common in the internal carotid $(n=18)$ than in the vertebral arteries $(n=6)$. All but one patient had intracranial embolus. IAT techniques included thrombectomy $(n=19)$, IA thrombolysis $(n=17)$, stent $(n=14)$, and angioplasty $(n=7)$. Outcomes included favorable 90 day mRS score of $0-2$ in $63 \%, 4$ deaths, 1 sICH, and 3 procedural complications. After adjustment, favorable mRS in our target population was similar to comparison populations: (a) in CeAD, IAT versus no IAT (OR 0.62, $p=0.56)$; (b) In CeAD, IAT versus IVT alone (OR 1.32, $p=0.79$ ); and (c) IAT in CeAD versus nonCeAD mechanism of AIS (OR 0.58, $\mathrm{p}=0.34$ ).

Conclusions IAT is a valid alternative therapeutic option for AIS caused by CeAD due to the low complication rate and excellent outcomes observed in this study.

\section{INTRODUCTION}

Cervical artery dissection (CeAD) is a recognized cause of acute ischemic stroke (AIS), characterized by intramural hematoma produced by subintimal tear of the carotid and vertebral arterial walls. ${ }^{1}$ $\mathrm{CeAD}$ is uncommon in the general stroke population accounting for just $2-2.5 \%$ of all AIS, ${ }^{2}$ but is a major cause of stroke in the young, responsible for $10-25 \%$ of all AIS. ${ }^{3} 4$

Patients presenting with CeAD are traditionally managed with antithrombotic therapy, including anticoagulants and antiplatelet aggregation agents. ${ }^{5}$
There is debate as to whether anticoagulation is superior to antiplatelet aggregation agents; ${ }^{16}$ the findings from the first published randomized controlled trial comparing antithrombotic therapies found no difference in efficacy, although this pilot trial's outcomes were rare occurrences. ${ }^{7}$ CeAD should not be considered a contraindication to acute treatment with intravenous thrombolysis (IVT) because IVT has been shown to be beneficial across all causes of stroke in inducing recanalization of the thrombosis and distal emboli.

Intra-arterial treatment (IAT) may be a complementary option to IVT and antithrombotic therapy in patients with AIS due to CeAD. There are several case reports and small case series of IAT in patients with $\mathrm{CeAD}$, demonstrating the feasibility of this approach. ${ }^{8-23}$ However, the safety and efficacy of IAT with CeAD has not been formally studied. In light of the recent groundbreaking randomized controlled trials (RCTs) ${ }^{24-28}$ demonstrating the superiority of IAT over IVT in selected patients with AIS caused by proximal intracranial occlusion in the anterior circulation, our purpose was to examine the safety and efficacy of IAT for AIS caused by CeAD.

\section{DESIGN}

We retrospectively studied all consecutively admitted patients with AIS at our high volume comprehensive stroke center between 1 January 2010 and 31 May 2015. The study was investigator initiated and approved by the institutional review board with a waiver of subject informed consent.

The specific aims of this study were twofold: first, to describe the characteristics and outcomes of a large series of patients with CeAD treated with IAT $(n=24)$; second, to analyze the odds of favorable outcome following IAT in the CeAD population versus three comparison populations: (a) patients with CeAD not receiving IAT $(n=137)$, (b) patients with CeAD receiving IVT alone $(n=11)$, and (c) patients with a non-CeAD cause of AIS receiving IAT $(n=421)$.

\section{Outcomes and covariates}

The following demographics and clinical characteristics were described: age, gender, race, transfer status, type of dissection, area of occlusion, history/ etiology, presenting signs and symptoms, diagnostic modality, National Institutes of Health Stroke Scale (NIHSS), and treatment type. Outcomes included 
favorable 90 day modified Rankin Scale score $(\mathrm{mRS} \leq 2$ at 90 days or at discharge where 90 day mRS scores were unavailable, vs mRS 3-6), recanalization (defined as successful/unsuccessful for the cervical artery, and defined as successful by Thrombolysis in Cerebral Infarction (TICI) scores $2 b-3$ for the associated intracranial embolus), procedural complications, rate of symptomatic intracerebral hemorrhage (sICH) defined using European Cooperative Acute Stroke Study criteria, ${ }^{29} 30$ inhospital mortality, and median change in NIHSS (discharge-initial NIHSS).

\section{Statistical analysis}

Analyses were performed with SAS V.9.3. $\chi^{2}$ and Wilcoxon rank sum tests were used to analyze demographic and clinical differences for patients with CeAD treated with IAT with our three comparison populations. The outcomes of favorable 90 day mRS $0-2$, sICH, and mortality were univariately analyzed with Pearson and Fisher's exact $\chi^{2}$ tests. Favorable 90 day mRS 0-2 was also analyzed with multivariate stepwise logistic regression using entry and exit criteria of $p<0.20$. Change in NIHSS was analyzed with Wilcoxon rank sum tests.
Data are presented as median (IQR), per cent (n), and OR (95\% CI). Statistical significance for all analyses was set at $\mathrm{p}<0.05$.

\section{RESULTS}

\section{CeAD and IAT case series}

Among 161 patients with CeAD, 24 (15\%) were managed acutely with IAT (table 1 ). The population was primarily male (75\%), aged 52 years (42-59.5), with an NIHSS score of 13 (12-16). Internal carotid artery dissections (ICAD; $n=18,1$ bilateral) were more common than vertebral artery dissections (VAD; $\mathrm{n}=6,2$ bilateral). All but one patient had associated intracranial embolus. Dissections were idiopathic $(n=17)$, traumatic $(n=5)$, or cough related $(n=2)$. Signs and symptoms included hemiparesis $(n=23,96 \%)$, hemisensory loss $(n=16$, $67 \%)$, aphasia $(n=15,63 \%)$, neglect $(n=10,42 \%)$, dysarthria $(n=22,92 \%)$, facial palsy $(n=21,88 \%)$, and gaze palsy $(n=16$, $66 \%)$

The majority of patients had multiple IAT techniques, with a median of $2(2-3)$ techniques. IAT techniques included thrombectomy $(\mathrm{n}=19)$, IA thrombolysis $(\mathrm{n}=17)$, stenting $(\mathrm{n}=14)$, and

Table 1 Intra-arterial thrombolysis treated patients with acute ischemic stroke due to cervical artery dissection

\begin{tabular}{|c|c|c|c|c|c|c|c|c|c|c|}
\hline ID & $\begin{array}{l}\text { Age } \\
\text { (years) }\end{array}$ & Transfer & $\begin{array}{l}\text { Baseline } \\
\text { NIHSS }\end{array}$ & $\begin{array}{l}\text { CeAD } \\
\text { type }\end{array}$ & $\begin{array}{l}\text { Intracranial } \\
\text { thrombus }\end{array}$ & IVT & IAT procedures & $\begin{array}{l}\text { CeAD } \\
\text { recanalization }\end{array}$ & $\begin{array}{l}\text { Intracranial embolus } \\
\text { recanalization }\end{array}$ & $\begin{array}{l}\text { Discharge } \\
\mathrm{mRS}\end{array}$ \\
\hline 1 & $40-49$ & Yes & 0 & Vertebral & Yes & No & $\begin{array}{l}\text { IA lytic, thrombectomy, } \\
\text { stent }\end{array}$ & Successful & $\mathrm{TICl} 3$ & 6 \\
\hline 2 & $50-59$ & Yes & 12 & Carotid & Yes & Prior & $\begin{array}{l}\text { Angioplasty, IA lytic, } \\
\text { thrombectomy, stent }\end{array}$ & Successful & $\mathrm{TICl} 3$ & 3 \\
\hline 3 & $60-69$ & Yes & 19 & Carotid & Yes & Prior & Stent & Successful & $\mathrm{TICl} 3$ & 3 \\
\hline 4 & $40-49$ & Yes & 27 & Carotid & No & Prior & Stent & Successful & $\mathrm{N} / \mathrm{A}$ & 2 \\
\hline 5 & $60-69$ & Yes & 13 & Carotid & Yes & No & IA lytic, thrombectomy & Non-flow limiting & Unsuccessful & 3 \\
\hline 6 & $80-89$ & Yes & . & Vertebral & Yes & No & IA lytic, thrombectomy & Left occluded & $\mathrm{TICl} 3$ & 6 \\
\hline 7 & $40-49$ & Yes & . & Vertebral $^{*}$ & Yes & No & IA lytic, thrombectomy & Successful & $\mathrm{TICl} 3$ & 2 \\
\hline 8 & $50-59$ & Yes & 15 & Carotid & Yes & No & $\begin{array}{l}\text { Angioplasty, IA lytic, } \\
\text { thrombectomy, stent }\end{array}$ & Successful & $\mathrm{TICl} 2 \mathrm{~b}$ & 4 \\
\hline 9 & $70-79$ & No & 9 & Vertebral* $^{*}$ & Yes & No & $\begin{array}{l}\text { Angioplasty, IA lytic, } \\
\text { thrombectomy, stent }\end{array}$ & Successful & 2bt/Unsuccessful & 6 \\
\hline 10 & $50-59$ & Yes & 12 & Carotid & Yes & Prior & Thrombectomy, stent & Successful & $\mathrm{TICl} 3$ & 2 \\
\hline 11 & $40-49$ & No & 24 & Carotid & Yes & No & IA lytic, stent & Successful & $\mathrm{TICl} 3$ & 4 \\
\hline 12 & $50-59$ & Yes & 14 & Carotid & Yes & Yes & $\begin{array}{l}\text { Angioplasty, IA lytic, } \\
\text { thrombectomy, stent }\end{array}$ & Successful & $\mathrm{TICl} 2 \mathrm{~b}$ & 4 \\
\hline 13 & $40-49$ & No & 10 & Carotid & Yes & Yes & Thrombectomy, stent & Successful & $\mathrm{TICl} 2 \mathrm{~b}$ & 4 \\
\hline 14 & $30-39$ & Yes & 16 & Carotid & Yes & No & $\begin{array}{l}\text { IA lytic, stent, } \\
\text { thrombectomy }\end{array}$ & Left occluded & $2 b$ & 3 \\
\hline 15 & $40-49$ & Yes & 12 & Carotid & Yes & Prior & IA lytic, thrombectomy & Non-flow limiting & $\mathrm{TICl} 2 \mathrm{~b}$ & 1 \\
\hline 16 & $60-69$ & Yes & 11 & Carotid* & Yes & No & Thrombectomy & Successful & $\mathrm{TICl} 3$ & 3 \\
\hline 17 & $20-29$ & Yes & . & Vertebral & Yes & No & Thrombectomy & Left occluded & $2 b$ & 5 \\
\hline 18 & $60-69$ & Yes & 15 & Carotid & Yes & Prior & $\begin{array}{l}\text { IA lytic, thrombectomy, } \\
\text { stent }\end{array}$ & Successful & $\mathrm{TICl} 2 \mathrm{~b}$ & 4 \\
\hline 19 & $40-49$ & Yes & 33 & Vertebral & Yes & Prior & Thrombectomy & Successful & $\mathrm{TICl} 2 \mathrm{~b}$ & 3 \\
\hline 20 & $50-59$ & Yes & 6 & Carotid & Yes & No & $\begin{array}{l}\text { Angioplasty, IA lytic, } \\
\text { thrombectomy }\end{array}$ & Non-flow limiting & $\mathrm{TICl} 2 \mathrm{~b}$ & 4 \\
\hline 21 & $50-59$ & Yes & 23 & Carotid & Yes & No & $\begin{array}{l}\text { Angioplasty, IA lytic, } \\
\text { stent }\end{array}$ & Successful & $\mathrm{TICl} 3$ & 6 \\
\hline 22 & $50-59$ & No & 13 & Carotid & Yes & No & $\begin{array}{l}\text { Angioplasty, IA lytic, } \\
\text { stent }\end{array}$ & Successful & $\mathrm{TICl} 3$ & 3 \\
\hline 23 & $50-59$ & Yes & 15 & Carotid & Yes & Prior & IA lytic, thrombectomy & Non-flow limiting & $\mathrm{TICl} 2 \mathrm{~b}$ & 4 \\
\hline 24 & $30-39$ & Yes & 12 & Carotid & Yes & Prior & IA lytic, thrombectomy & Non-flow limiting & $\mathrm{TICl} 3$ & 4 \\
\hline
\end{tabular}

*Bilateral dissections.

tPatient No 9 had successful restoration of flow and then re-thrombosed 2 days later, with unsuccessful recanalization of re-thrombosis.

CeAD, cervical artery dissection; IAT, intra-arterial thrombolysis, IVT, intravenous thrombolysis; mRS, modified Rankin score; NIHSS, National Institutes of Health Stroke Scale; TICI

Thrombolysis in Cerebral Infarction. 
angioplasty $(n=7)$. Eleven patients were also treated with IV tissue plasminogen activator.

Regarding stents, 14 patients with at least 99\% CeAD occlusion were stented: 7 stents were placed before approaching the intracranial embolus; 4 stents were placed after approaching the intracranial embolus; patient No 14 was unsuccessfully stented both before and after recanalization of the middle cerebral artery (MCA); patient No 4 had an ICAD stent and did not have an associated intracranial embolus; and patient No 3 had an ICAD stent and did not have IAT performed on the intracranial embolus. More than one stent was required in 8 of 14 patients.

Favorable 90 day mRS 0-2 was achieved in 63\% (12/19) of patients. The median improvement in NIHSS was -9 points (IQR -11 to 1$)$. There were four deaths; none was attributable to IAT. There was $1 \mathrm{sICH}$ and 3 procedural complications (2 groin pseudoaneurysms, 1 catheter induced acute dissection).

Among the 18 ICADs, 6 internal carotid arteries were left untreated: 5 were non-flow limiting and 1 was left occluded with excellent intracranial collateral circulation following unsuccessful stenting of the right ICAD (table 1). The remaining 12 ICADs were successfully recanalized (100\% successful recanalization).

Seventeen of 18 ICADs had associated MCA occlusions; patient No 4 did not have an associated MCA occlusion but was stented due to inadequate collateral flow. Recanalization of the MCA was successful to TICI $2 \mathrm{~b}-3$ in 16 patients and was unsuccessful in 1 patient ( $94 \%$ successful recanalization). Patient No 3 was technically successful with prompt restoration of flow to the intracranial circulation after the ICAD was stented, although the small MCA branch occlusions were not approached.

Among the six VADs, two vertebral arteries were left occluded because the risk of opening it was greater than the perceived benefit of revascularization. The remaining four VADs were successfully recanalized (100\%) (table 1).

All six VADs had a basilar artery occlusion; recanalization of the basilar artery was $100 \%$ successful to TICI $2 \mathrm{~b}-3$ (table 1). In the two VADs where the vertebral artery was left untreated, the basilar artery embolus was extracted through the contralateral patent vertebral artery. In patient No 9, the vertebral artery re-thrombosed 2 days after the initial restoration of flow and the vertebral artery was unsuccessfully recanalized.

\section{Outcomes analysis}

Overall there were 3671 patients with AIS. There were 445 patients treated with IAT, including 421 patients with a non-CeAD mechanism of AIS and 24 patients with a CeAD mechanism.
Thirty-five patients with CeAD were acutely treated with IAT $(n=24 ; 11$ with and 13 without IVT) or IVT only $(n=11)$ (figure 1). The remaining 125 patients with CeAD were acutely treated with either anticoagulation or antiplatelet therapy.

First we analyzed patients with CeAD who received IAT versus those who did not receive IAT. There were significant differences in demographics, clinical characteristics, and outcomes (table 2). In particular, patients with CeAD who were treated with IAT had significantly more severe stroke compared with those not receiving IAT (median NIHSS 13 vs 3, p<0.001). As a result, unadjusted outcomes were significantly worse in patients treated with IAT than patients who did not receive IAT, with the exception of a greater improvement in NIHSS ( -9 vs $1, p=0.02)$. However, after adjustment for baseline NIHSS and age, the odds of a favorable 90 day mRS 0-2 were not significantly different for patients receiving IAT versus Those not receiving IAT (OR $0.62(0.12-3.14), \mathrm{p}=0.56)$ (figure 2 ).

Next we analyzed patients with CeAD treated with IAT versus IVT alone. There were no significant differences between groups in demographics, clinical characteristics, or unadjusted outcomes, except for patients receiving IAT who had more severe stroke than those receiving IVT alone (median NIHSS 13 vs 10, $\mathrm{p}=0.04$ ) (table 2). After adjustment for baseline NIHSS, there was no difference in the odds of a favorable 90 day mRS 0-2 with IAT versus IVT alone (OR $1.32(0.16-10.72), p=0.79)$ (figure 2).

Finally, we analyzed outcomes in 445 patients receiving IAT: 24 with CeAD and 421 with another cause of AIS. Patients with CeAD were younger (median age 52 vs $70, \mathrm{p}<0.001$ ) with less severe stroke (median NIHSS 13 vs $18, \mathrm{p}=0.03$ ) than those with another cause of AIS (table 2). Despite these differences, unadjusted outcomes were similar by AIS cause (table 2). After adjustment for baseline NIHSS and age, there was no difference in the odds of a favorable 90 day mRS 0-2 with CeAD versus another cause of AIS (OR $0.58(0.19-1.78), \mathrm{p}=0.34)$ (figure 2).

\section{DISCUSSION}

Groundbreaking findings of several recently published RCTs demonstrated the superiority of IAT over standard medical therapy, resulting in updated American Heart Association/ American Stroke Association guidelines recommending endovascular treatment in selected patients with AIS. ${ }^{31}$ The role of IAT in patients with CeAD is empirically demonstrated because there are no controlled trials; data are confined to 16 published case series, ${ }^{8-23}$ the largest of which reported on 26 patients with CeAD and IAT, ${ }^{15} 32$ and two systematic reviews by Pham and Zinkstok. ${ }^{33} 34$ The review by Pham et al ${ }^{33}$ reported a 99\%
Figure 1 Flowchart of patients with acute ischemic stroke, by mechanism and type of treatment. ${ }^{a}$ Analysis of comparison populations are noted. CeAD, cervical artery dissection; IAT, intra-arterial thrombolysis, IVT, intravenous thrombolysis.

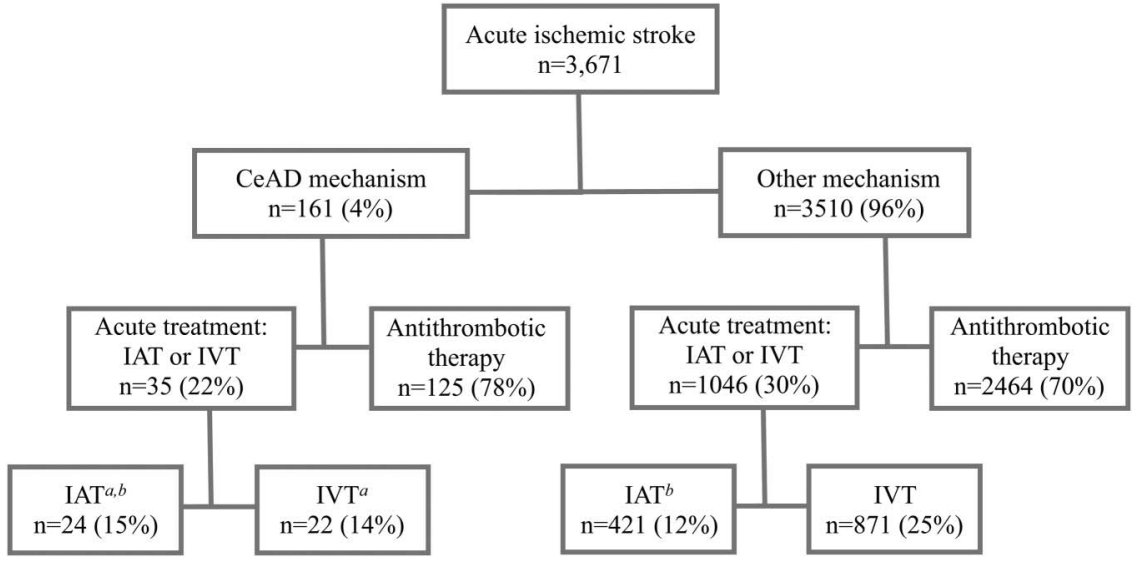


Ischemic stroke

Table 2 Clinical and demographic characteristics and unadjusted outcomes

\begin{tabular}{|c|c|c|c|c|}
\hline & CeAD and IAT $(n=24)$ & CeAD, No IAT $(n=137)$ & CeAD, IVT alone $(n=11)$ & Non-CeAD mechanism $(n=421)$ \\
\hline Age (years) & $52(43-60)$ & $44(35-54) \dagger$ & $52(37-59)$ & $70(61-79) \ddagger$ \\
\hline Female sex & $25.0(6)$ & $41.6(57)^{*}$ & $36.4(4)$ & $54.16(228) \ddagger$ \\
\hline White race & $83.3(20)$ & $65.0(89)$ & $100.0(11)$ & 77.4 (326) \\
\hline Transferred in & $83.3(20)$ & $69.3(95)$ & 72.7 (8) & $71.7(302)$ \\
\hline IV tPA & $45.8(11)$ & $8.0(11) \ddagger$ & $100(11)$ & $58.4(246)$ \\
\hline Initial NIHSS & $13(12-16)$ & $3(1-7) \neq$ & $10(7-12) \dagger$ & $18(13-22) \dagger$ \\
\hline Discharge NIHSS & $6(3-13)$ & $1(0-3) \ddagger$ & $9(3-16.5)$ & $1(0-3) \ddagger$ \\
\hline Change in NIHSS & $-9(-11-1)$ & $1(-3-0) \dagger$ & $-4(-5.5-5)$ & $-10(-15-5)$ \\
\hline Discharge $\mathrm{mRS}$ & $4(3-4)$ & $2(1-3)$ & $3.5(3-4)$ & $2(1-3) \ddagger$ \\
\hline 90 day $\mathrm{mRS} 0-2 \S$ & $65.0(13 / 20)$ & $93.0(93) \ddagger$ & $60.0(3 / 5)$ & $53.7(145 / 270)$ \\
\hline Symptomatic ICH & $4.0(1 / 25)$ & $0(0)^{*}$ & $0.0(0 / 11)$ & $6.4(27 / 421)$ \\
\hline Inhospital mortality & $16.0(4 / 25)$ & $3.7(5) \dagger$ & $9.1(1 / 11)$ & $16.4(69 / 421)$ \\
\hline
\end{tabular}

Values are $\%(\mathrm{n})$ or median (IQR).

Change in NIHSS: discharge NIHSS-initial NIHSS (negative values indicate improvement).

Pearson $\chi^{2} p$ value versus CeAD and IAT.

${ }^{*}<0.15$.

$\dagger<0.05$.

$\ddagger<0.01$.

§Defined as $\mathrm{mRS} 0-2$ at 90 days or discharge, or discharge home.

CeAD, cervical artery dissection; IAT, intra-arterial thrombolysis; ICH, intracerebral hemorrhage; IVT, intravenous thrombolysis; IV tPA, intravenous tissue plasminogen activator; mRS,

modified Rankin Scale; NIHSS, National Institutes of Health Stroke Scale.

technical success rate and $1.3 \%$ complication rate with IAT in 140 patients with CeAD; data on mRS were not summarized. The systematic review by Zinkstok et $a l^{34}$ reported safety and efficacy of thrombolysis (IAT and IVT) in 180 patients with CeAD, of whom 59 received IAT. Overall rates were $3 \%$ sICH, $9 \%$ mortality, and $56 \%$ with $\mathrm{mRS} \leq 2$. These rates are similar to our case series of CeAD patients treated with IAT, with $4 \%$ sICH, $17 \%$ mortality, and $63 \%$ with $\mathrm{mRS} \leq 2$.

This study also provides class III evidence that the rate of functional independence, defined as mRS 0-2 at 90 days, was similar for our target population of patients with CeAD treated with IAT as the rates observed in our three comparison populations, after adjustment for relevant covariates (namely stroke severity and age). However, caution must be taken when interpreting these findings because the study was not powered to detect significant differences between the groups due to small sample size. First, we examined IAT versus no IAT in 161 patients with $\mathrm{CeAD}$, reporting non-significant adjusted odds of

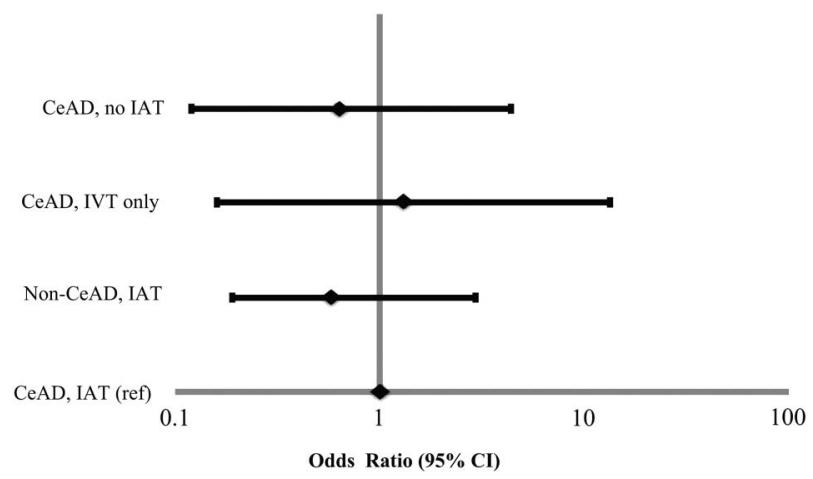

Figure 2 Adjusted odds of a favorable 90 day modified Rankin Scale score (mRS) (defined as mRS 0-2 at 90 days or discharge, or discharge home). Point estimates are noted with a diamond; estimates to the right of 1.0 favor the referent population. CeAD, cervical artery dissection; IAT, intra-arterial thrombolysis, IVT, intravenous thrombolysis. functional independence in favor of no IAT (OR 0.62, 0.123.14). Only one other study performed a similar analysis: Engelter et al used the Cervical Artery Dissection and Ischemic Stroke Patients (CADISP) registry to examine patients with CeAD and compared any thrombolysis $(n=68,9$ of whom had IAT and 55 had IVT) with no treatment $(n=548) .{ }^{35}$ In their study, the odds of functional independence were virtually identical between groups, both after adjustment (OR 0.95, 0.452.00) and after propensity matching (OR 1.00, 0.49-2.00). Second, we examined patients with CeAD and compared IAT versus IVT alone, reporting adjusted odds of functional independence of 1.32 (0.16-10.72). We identified two meta-analyses that performed a similar analysis, both showing nearly identical odds of functional independence to our study, all nonsignificantly favoring IAT to IVT alone. Engelter's 2015 meta-analysis examined 111 patients (32 received endovascular therapy) and reported an OR of $1.41(0.45-3.45) .{ }^{1}$ Zinkstok et al examined 180 patients (59 received IAT), reporting an OR of $1.45(0.61-3.45) .^{1}{ }^{34}$ Lastly, we compared IAT in patients with CeAD versus another cause of AIS. Two meta-analyses examined IAT in CeAD versus a non-CeAD mechanism. The first meta-analysis did not report efficacy outcomes but rather showed the sICH rate was no different following IAT in patients with CeAD compared with other causes of AIS identified via the Safe Implementation of Thrombolysis in Stroke-Monitoring Study (SITS-MOST) registry (1.9\% vs $1.7 \%) .{ }^{36}$ While our rates of sICH were higher, we also found no significant difference in sICH rates between groups, at $4 \%$ versus $6.4 \%$. The second meta-analysis found no difference in the rates of functional independence after IAT in patients with CeAD compared with other causes of AIS identified via the Safe Implementation of Thrombolysis in Stroke-International Stroke Thrombolysis (SITS-ISTR) registry (56\% vs 52\%). ${ }^{34}$ Our reported rates of functional independence after IAT in those with versus Those without CeAD mechanism were comparable $(65 \%$ and $54 \%$, respectively).

Our results, taken together with the published meta-analyses, suggest three key findings: (1) IAT appears to be as safe and effective when used in patients with ischemic stroke due to 
CeAD compared with other causes; (2) IAT may be favored over IVT alone based on the direction of the adjusted ORs of $1.32,1.41$, and 1.45 across published studies (the CIs for all ORs were wide, which can be partially explained by the small sample sizes); and (3) despite the IAT group being a select group with more severe baseline deficits and presence of occlusions, the adjusted odds of achieving functional independence was similar in patients with CeAD treated with IAT to those not treated with IAT.

Our comprehensive stroke center has an active interventional neuroradiology program, with approximately 150 IAT procedures performed in the past year. Our stroke center does not have explicit guidelines, but as a general rule patients are treated with IAT if there is distal embolus or thrombus extension or if there is a decline despite receiving standard medical therapy. CeAD is not considered a contraindication to treatment; approximately $15 \%$ of the CeAD population were treated with IAT, which is similar to $12 \%$ treatment in the broader AIS population. Patients with CeAD who were treated with IAT were likely selected because of the presence of large deficits and MCA or basilar artery occlusions.

The primary limitation to this analysis was that this study was retrospective and carries with it the limitations of such a study design, including reliance on existing medical records and lack of controls. Second, this study was conducted at a single center and, despite reporting a large series of CeAD, the subgroups for analysis were small. While our analysis was performed in a smaller sample size than the published meta-analyses and the study using the CADISP registry, we were able to control for potentially confounding factors, such as population and treating physician and hospital, in addition to stroke severity.

\section{CONCLUSION}

These data suggest IAT is an alternative therapeutic option for AIS due to CeAD. Outcomes appear to be similar to those presented in large RCTs with stroke from all causes, and to the comparison populations at our high volume comprehensive stroke center. Following the major advances in IAT for AIS in the general population, IAT should be considered in patients with AIS due to CeAD. Given the small numbers in our series and the paucity of data in the literature, we encourage others to present their experience with IAT in the CeAD population. We believe randomized trials of IAT in CeAD are appropriate and timely, particularly in patients with intracranial occlusions.

Contributors All authors gave final approval of the published version and agree to be accountable for all aspects of the work in ensuring that questions related to the accuracy or integrity of any part of the work are appropriately investigated and resolved. Specifically, JJ contributed to conception and design, acquisition and interpretation of the data, and drafting the manuscript. KS contributed to conception and design, analysis and interpretation of the data, and drafting the manuscript. DF contributed to acquisition and interpretation of the data and critical revision. DL contributed to design of the study and critical revision. $\mathrm{KMCC}$ contributed to acquisition of the data and critical revision. JW contributed to interpretation of the data and critical revision. MW contributed to acquisition of the data and critical revision. RB contributed to interpretation of the data and critical revision. DB-O contributed to interpretation of data and critical revision.

Competing Interests DF reports a consulting relationship with MicroVention, Siemens, Penumbra, Stryker, and Codman. DF owns options to purchase Penumbra equity. JW is a speaker for Genentech. MW reports a consulting relationship with Clinical Data Management and Lombardi Hill, and is a speaker for Activase. RB is a speaker for Penumbra.

Ethics approval The study was approved by HealthOne IRB.

Provenance and peer review Not commissioned; externally peer reviewed.

Data Sharing statement Anonymized data and statistical code are available from the corresponding author (dbaror@ampiopharma.com).
Open Access This is an Open Access article distributed in accordance with the Creative Commons Attribution Non Commercial (CC BY-NC 4.0) license, which permits others to distribute, remix, adapt, build upon this work non-commercially, and license their derivative works on different terms, provided the original work is properly cited and the use is non-commercial. See: http://creativecommons.org/ licenses/by-nc/4.0/

\section{REFERENCES}

1 Engelter ST, Traenka C, Von Hessling A, et al. Diagnosis and treatment of cervical artery dissection. Neurol Clin 2015;33:421-41.

2 Debette S, Leys D. Cervical-artery dissections: predisposing factors, diagnosis, and outcome. Lancet Neurol 2009;8:668-78.

3 Leys $\mathrm{D}$, Bandu L, Hénon $\mathrm{H}$, et al. Clinical outcome in 287 consecutive young adults (15 to 45 years) with ischemic stroke. Neurology 2002;59:26-33.

4 Yesilot Barlas N, Putaala J, Waje-Andreassen U, et al. Etiology of first-ever ischaemic stroke in European young adults: the 15 cities young stroke study. Eur $J$ Neurol 2013;20:1431-9.

5 Sacco RL, Adams R, Albers G, et al. Guidelines for prevention of stroke in patients with ischemic stroke or transient ischemic attack: a statement for healthcare professionals from the American Heart Association/American Stroke Association Council on Stroke: co-sponsored by the Council on Cardiovascular Radiology and Intervention: the American Academy of Neurology affirms the value of this guideline. Stroke 2006;37:577-617.

6 Arnold M, Fischer U, Bousser MG. Treatment issues in spontaneous cervicocephalic artery dissections. Int I Stroke 2011;6:213-18.

7 Markus HS, Hayter E, Levi C, et al, CADISS trial investigators. Antiplatelet treatment compared with anticoagulation treatment for cervical artery dissection (CADISS): a randomised trial. Lancet Neurol 2015;14:361-7.

8 Arnold M, Nedeltchev K, Sturzenegger M, et al. Thrombolysis in patients with acute stroke caused by cervical artery dissection: analysis of 9 patients and review of the literature. Arch Neurol 2002:59:549-53.

9 Assadian A, Senekowitsch C, Rotter R, et al. Long-term results of covered stent repair of internal carotid artery dissections. J Vasc Surg 2004;40:484-7.

10 Cohen JE, Leker RR, Gotkine M, et al. Emergent stenting to treat patients with carotid artery dissection: clinically and radiologically directed therapeutic decision making. Stroke 2003;34:e254-7.

11 Dabitz R, Triebe S, Leppmeier U, et al. Percutaneous recanalization of acute internal carotid artery occlusions in patients with severe stroke. Cardiovasc Intervent Radiol 2007;30:34-41.

12 Edgell RC, Abou-Chebl A, Yadav JS. Endovascular management of spontaneous carotid artery dissection. J Vasc Surg 2005;42:854-60; discussion 860

13 Fava M, Meneses L, Loyola S, et al. Carotid artery dissection: endovascular treatment. Report of 12 patients. Catheter Cardiovasc Interv 2008;71:694-700.

14 Fields JD, Lutsep HL, Rymer MR, et al. Endovascular mechanical thrombectomy for the treatment of acute ischemic stroke due to arterial dissection. Interv Neuroradiol 2012;18:74-9.

15 Kadkhodayan Y, Jeck DT, Moran CJ, et al. Angioplasty and stenting in carotid dissection with or without associated pseudoaneurysm. AJNR Am J Neuroradiol 2005:26:2328-35.

16 Lekoubou A, Cho TH, Nighoghossian N, et al. Combined intravenous recombinant-tissular plasminogen activator and endovascular treatment of spontaneous occlusive internal carotid dissection with tandem intracranial artery occlusion. Eur Neurol 2010;63:211-14.

17 Malek AM, Higashida RT, Phatouros CC, et al. Endovascular management of extracranial carotid artery dissection achieved using stent angioplasty. AJNR Am J Neuroradiol 2000;21:1280-92.

18 Vergouwen MD, Beentjes PA, Nederkoorn PJ. Thrombolysis in patients with acute ischemic stroke due to arterial extracranial dissection. Eur J Neurol 2009;16:646-9.

19 Ahmad HA, Gerraty RP, Davis SM, et al. Cervicocerebral artery dissections. J Accid Emerg Med 1999;16:422-4.

20 Cerrato P, Berardino M, Bottacchi E, et al. Vertebral artery dissection complicated by basilar artery occlusion successfully treated with intra-arterial thrombolysis: three case reports. Neurol Sci 2008;29:51-5.

21 Mourand I, Brunel $\mathrm{H}$, Vendrell JF, et al. Endovascular stent-assisted thrombolysis in acute occlusive carotid artery dissection. Neuroradiology 2010;52:135-40.

22 Ohta H, Natarajan SK, Hauck EF, et al. Endovascular stent therapy for extracranial and intracranial carotid artery dissection: single-center experience. J Neurosurg 2011:115:91-100

23 Saeed AB, Shuaib A, Al-Sulaiti G, et al. Vertebral artery dissection: warning symptoms, clinical features and prognosis in 26 patients. Can J Neurol Sci 2000;27:292-6.

24 Berkhemer OA, Fransen PS, Beumer D, et al. A randomized trial of intraarterial treatment for acute ischemic stroke. N Engl J Med 2015;372:11-20.

25 Campbell BC, Mitchell PJ, Kleinig TJ, et al. Endovascular therapy for ischemic stroke with perfusion-imaging selection. N Eng/ J Med 2015;372:1009-18.

26 Goyal M, Demchuk AM, Menon BK, et al. Randomized assessment of rapid endovascular treatment of ischemic stroke. N Engl J Med 2015;372:1019-30 
27 Jovin TG, Chamorro A, Cobo E, et al. Thrombectomy within 8 hours after symptom onset in ischemic stroke. N Engl J Med 2015;372:2296-306.

28 Saver JL, Goyal M, Bonafe A, et al. Stent-retriever thrombectomy after intravenous t-PA vs. t-PA alone in stroke. N Engl J Med 2015;372:2285-95.

29 Berger C, Fiorelli M, Steiner T, et al. Hemorrhagic transformation of ischemic brain tissue: asymptomatic or symptomatic? Stroke 2001;32:1330-5.

30 Trouillas P, von Kummer R. Classification and pathogenesis of cerebral hemorrhages after thrombolysis in ischemic stroke. Stroke 2006;37:556-61.

31 Powers WJ, Derdeyn CP, Biller J, et al. 2015 American Heart Association/American Stroke Association Focused Update of the 2013 Guidelines for the Early Management of Patients With Acute Ischemic Stroke Regarding Endovascular Treatment: A Guideline for Healthcare Professionals From the American Heart Association/American Stroke Association. Stroke 2015;46:3020-35.
32 Ahlhelm F, Benz RM, Ulmer S, et al. Endovascular treatment of cervical artery dissection: ten case reports and review of the literature. Interv Neurol 2013;1:143-50.

33 Pham MH, Rahme RJ, Arnaout 0, et al. Endovascular stenting of extracranial carotid and vertebral artery dissections: a systematic review of the literature. Neurosurgery 2011;68:856-66; discussion 866.

34 Zinkstok SM, Vergouwen MDI, Engelter ST, et al. Safety and functional outcome of thrombolysis in dissection-related ischemic stroke: a meta-analysis of individual patient data. Stroke 2011;42:2515-20.

35 Engelter ST, Dallongeville J, Kloss M, et al. Thrombolysis in cervical artery dissection - data from the Cervical Artery Dissection and Ischaemic Stroke Patients (CADISP) database. Eur J Neurol 2012;19:1199-206.

36 Menon R, Kerry S, Norris JW, et al. Treatment of cervical artery dissection: a systematic review and meta-analysis. J Neurol Neurosurg Psychiatry 2008;79:1122-7. 